\title{
Discovery of Harmaline as a Potent Inhibitor of Sphingosine Kinase-1: A Chemopreventive Role in Lung Cancer
}

Sonam Roy, Taj Mohammad, Preeti Gupta, Rashmi Dahiya, Shahnaz Parveen, Suaib

Luqman, Gulam Mustafa Hasan, and Md. Imtaiyaz Hassan*

*Correspondence: mihassan@jmi.ac.in (M. I. H)

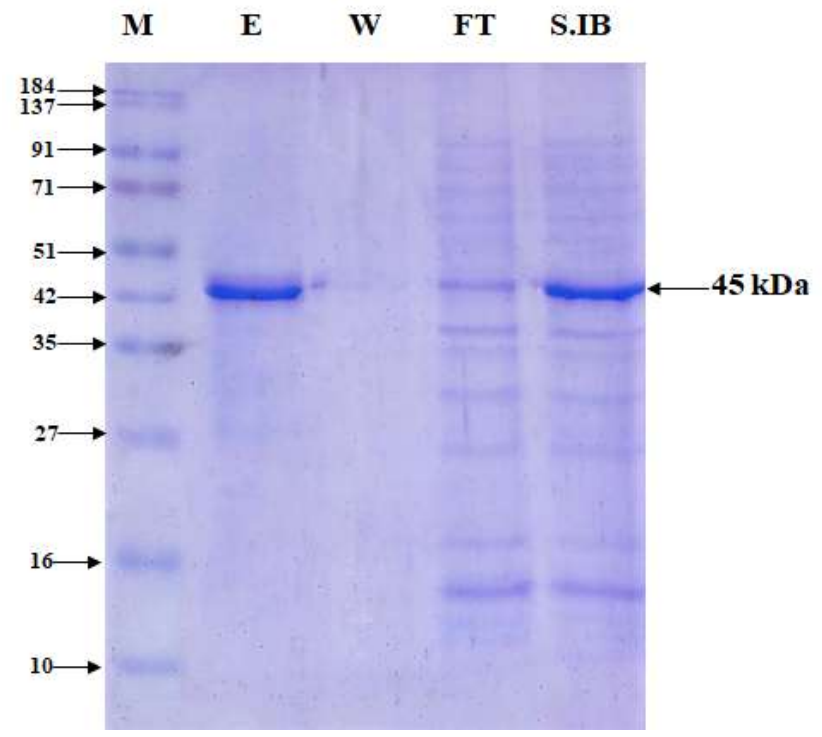

Figure S1. SDS-PAGE profile showing single band of SphK1 at approximately $45 \mathrm{kDa}$. The lanes S.IB, FT, W and E represent the solubilised inclusion bodies, flow through (unbound proteins), wash and final eluted SphK1. 


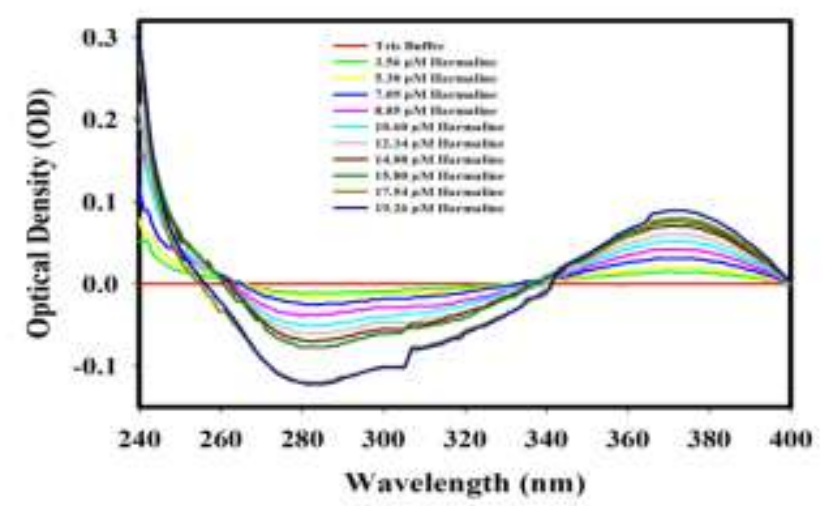

Figure S2. Absorption spectrum of increasing concentration of harmaline (3.56-19.26 $\mu \mathrm{M})$ in $20 \mathrm{mM}$ Tris, 100 $\mathrm{mM} \mathrm{NaCl}$ buffer ( $\mathrm{pH} \mathrm{8.0)}$ ). Harmaline absorbs in the range of 342-400 $\mathrm{nm}$ while no absorption is observed at $280 \mathrm{~nm}$. After $260 \mathrm{~nm}$, sharp increase in the absorbance of harmaline can be seen.
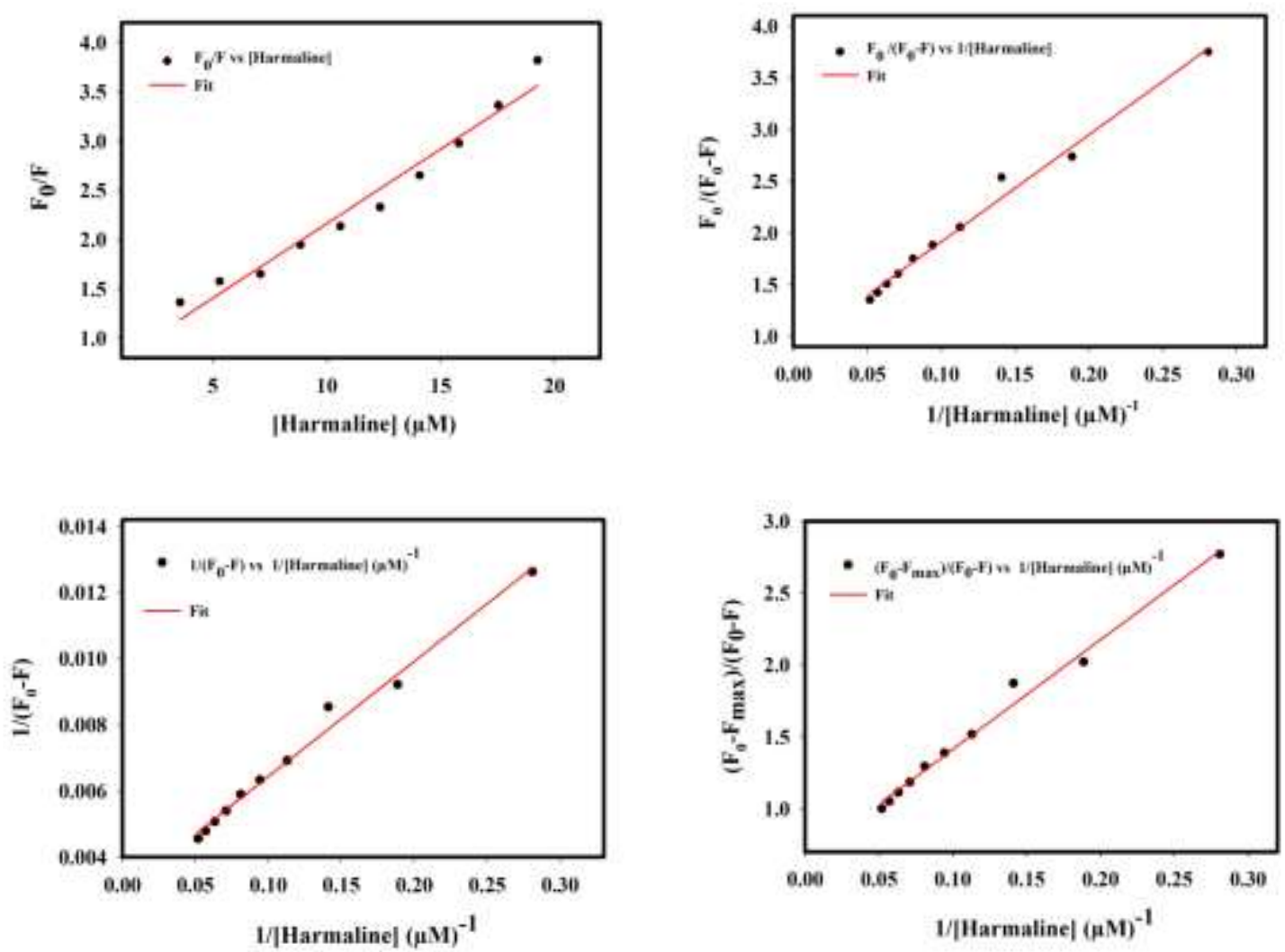

Figure S3. (A) Stern -Volmer plot for quenching of SphK1 $(4 \mu \mathrm{M}, \mathrm{pH} 8.0)$ by harmaline at $25^{\circ} \mathrm{C}$. (B) Modified Stern-Volmer plot for the estimation of the fraction of accessible fluorophore (SphK1). (C) The modified SternVolmer (Lineweaver-Burk) curve of harmaline quenching SphK1 fluorescence at $25^{\circ} \mathrm{C}$. (D) Plot of Johansson equation. 

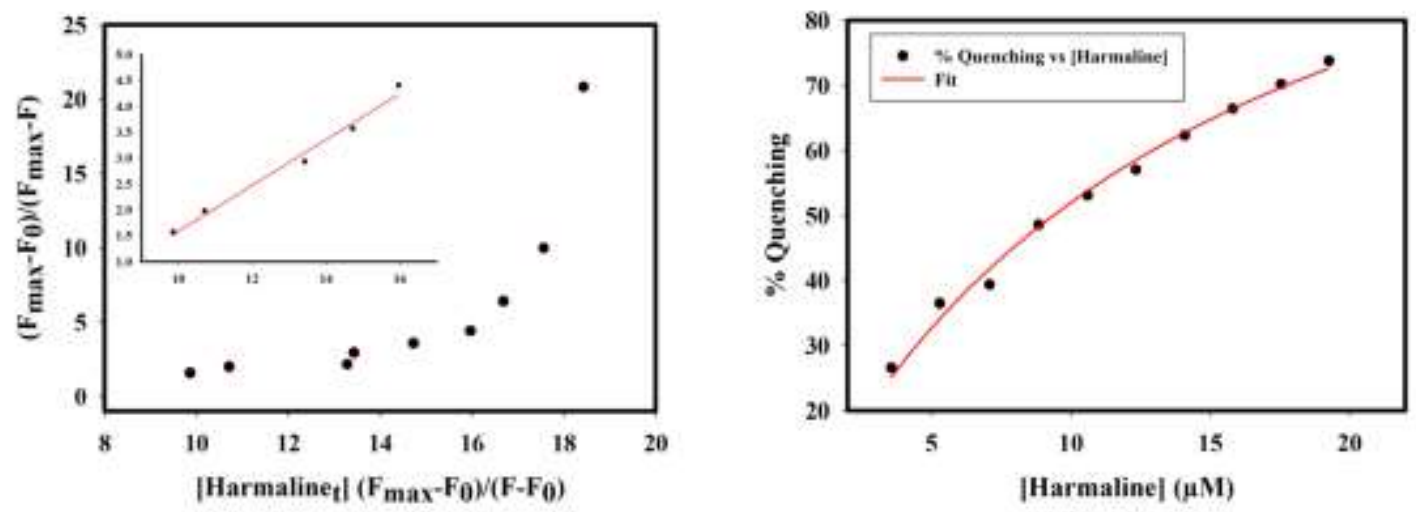

Figure S4. (A) The Scatchard plot of Harmaline quenching SphK1 fluorescence at $25{ }^{\circ} \mathrm{C}$. (B) Langmuir binding Fitting curve (one site saturation) of the quenching of the fluorescence of SphK1 by Harmaline.

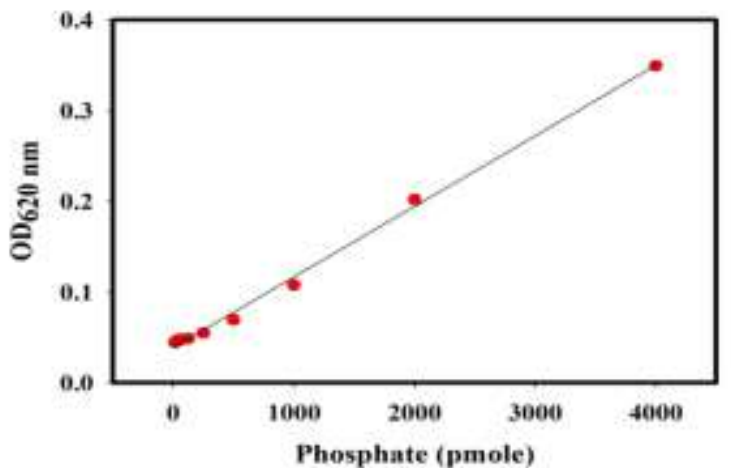

Figure S5. Standard phosphate hydrolysis curve showing the quantity of phosphate measured. 

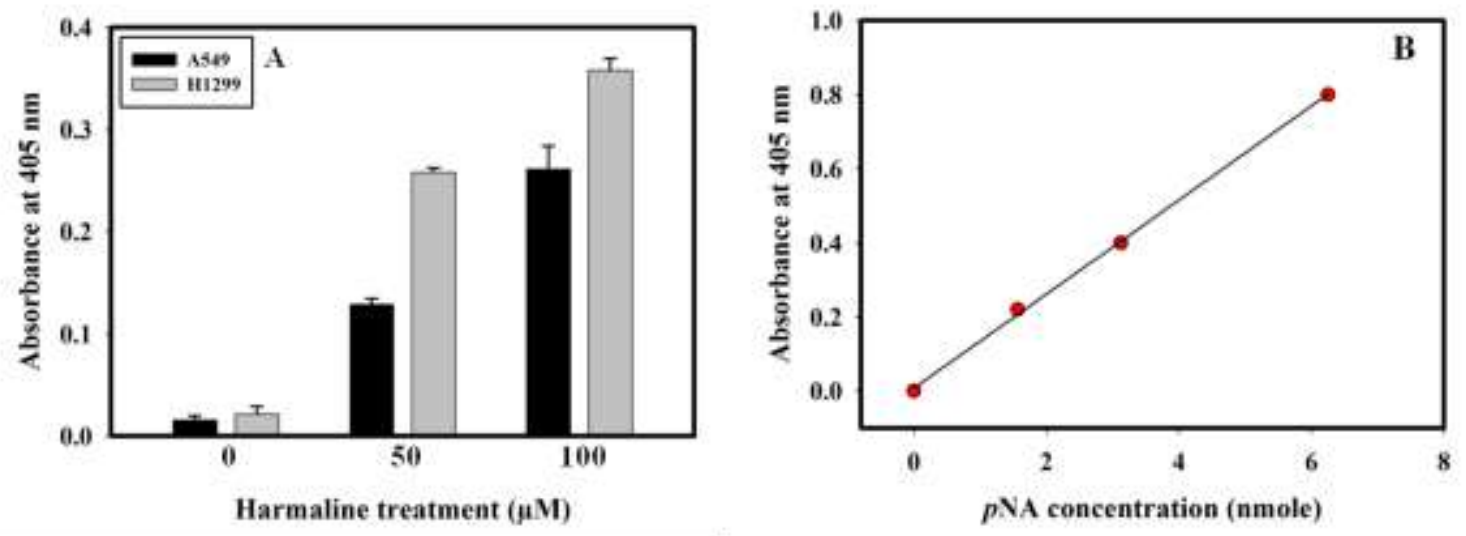

Figure S6. (A) An increase in the absorbance at $405 \mathrm{~nm}$ (corresponding to free pNA liberated) for both A549 and H1299 cells was recorded after treatment with 0,50 and $100 \mu \mathrm{M}$ of harmaline for $24 \mathrm{~h}$. (B) Standard $p$ NA curve that depicts the absorbance of free $p \mathrm{NA}$ at different concentrations.

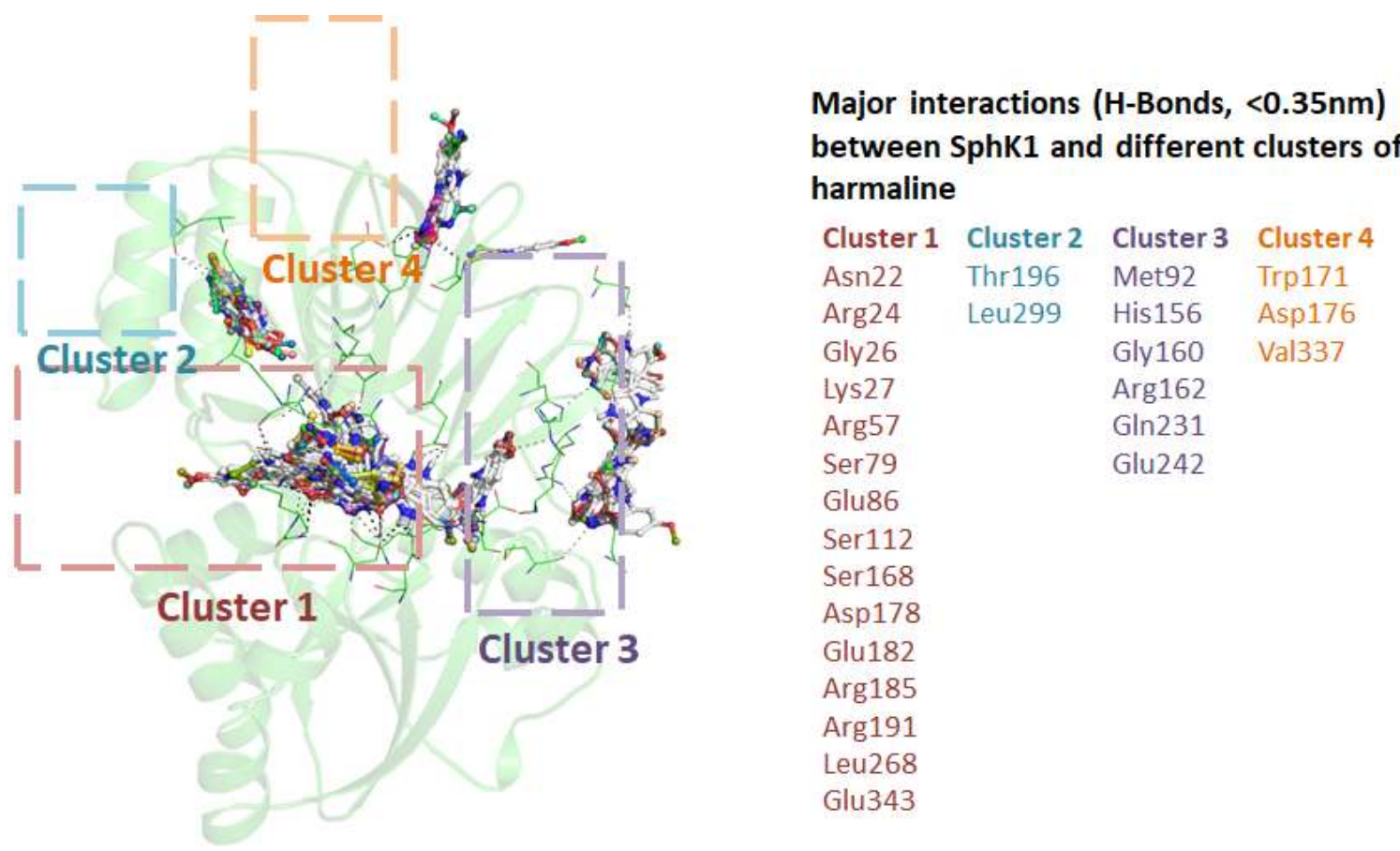

Figure S7. Plausible docking sites for harmaline on SphK1. Cartoon representation of SphK1 showing 4 plausible binding sites for harmaline docked conformers. Dotted boxes showing the clusters of harmaline conformers ranked based on their binding energy and number of conformers binding at the same site. +Cluster 1 has the lowest binding energy (thus highest affinity) and highest number of docked conformations and so on. Average binding affinity for Cluster 1 , Cluster 2, Cluster 3, and Cluster 4 was estimated as $-7.9,-7.4,-6.8$, and $-6.4 \mathrm{kcal} / \mathrm{mol}$, respectively. Major hydrogen bonding interactions between $\mathrm{SphK} 1$ and different clusters of harmaline are also shown on the right side.

Table S1.The $K_{\mathrm{a}}$ and $n$ of harmaline and SphK1 calculated using different models. 


\begin{tabular}{|l|l|l|l|}
\hline Equation & $\boldsymbol{K}_{\mathbf{a}}$ & $\boldsymbol{R}$ & $\boldsymbol{R} / \boldsymbol{R}^{2}$ \\
\hline$(1)$ & $1.5 \times 10^{5} \mathrm{M}^{-1}$ & - & $0.97 / 0.98$ \\
\hline$(2)$ & $8.5 \times 10^{4} \mathrm{M}^{-1}$ & 1.14 & $0.99 / 0.99$ \\
\hline$(3)$ & $7.1 \times 10^{5} \mathrm{M}^{-1}$ & 1.2 & $0.99 / 0.98$ \\
\hline$(4)$ & $8.62 \times 10^{4} \mathrm{M}^{-1}$ & - & $0.99 / 0.99$ \\
\hline$(5)$ & $1.3 \times 10^{5} \mathrm{M}^{-1}$ & - & $0.99 / 0.98$ \\
\hline$(6)$ & $4.4 \times 10^{5} \mathrm{M}^{-1}$ & 1.6 & $0.99 / 0.98$ \\
\hline$(7)$ & $6.9 \times 10^{4} \mathrm{M}^{-1}$ & 1 & $0.99 / 0.99$ \\
\hline
\end{tabular}

